\title{
Application of Tube Dynamics to Non-Statistical Reaction Processes*
}

\author{
F. Gabern ${ }^{1,2}$, W. S. Koon ${ }^{2}$, J. E. Marsden ${ }^{2}$, S. D. Ross ${ }^{3}$, and T. Yanao ${ }^{2}$ \\ 1 Departament de Matemàtica Aplicada I, Universitat Politècnica de Catalunya, \\ Av. Diagonal 647, 08028 Barcelona, Spain \\ 2 Control and Dynamical Systems, California Institute of Technology, MC 107-81, \\ Pasadena, CA 91125, USA \\ 3 Department of Aerospace and Mechanical Engineering, \\ University of Southern California, RRB 217, Los Angeles, CA 90089-1191, USA
}

Received November 14, 2005; accepted December 1, 2005

Published online May 31, 2006; C Springer-Verlag 2006

\begin{abstract}
A technique based on dynamical systems theory is introduced for the computation of lifetime distributions and rates of chemical reactions and scattering phenomena, even in systems that exhibit non-statistical behavior. In particular, we merge invariant manifold tube dynamics with Monte Carlo volume determination for accurate rate calculations. This methodology is applied to a three-degree-of-freedom model problem and some ideas on how it might be extended to higher-degree-of-freedom systems are presented.
\end{abstract}

\section{Introduction}

Chemical reaction rates are usually computed using statistical methods, such as Rice-Ramsperger-Kassel-Marcus (RRKM) theory, also known as transition state theory (TST). TST is based on the identification of a transition state (TS) between regions of phase space that correspond to either "reactants" or "products". TST yields rates based on a local study of the TS as well as the assumption that the phase space in each region is structureless. These values can be several orders of magnitude off of experimental values [1]. Despite its shortcomings, TST has been a workhorse of the chemistry community for decades. However, it is now well known that while the structureless assumption is useful in many situations, in general these regions (often defined by potential wells) are not structureless.

\footnotetext{
* Article based on the presentation by F. Gabern at the Fourth Workshop on the Dynamics and Structure of Critically Stable Quantum Few-Body Systems, MPIPKS, Dresden, Germany, 2005
} 
Many attempts have been made to remedy this situation. De Leon et al. [2] attempted to extend the local picture near the TS in two-degree-of-freedom (dof) systems to a more global one and developed reaction island theory using cylindrical manifolds. Komatsuzaki and Berry [3] used perturbative methods to study the transition near the saddle region. Uzer et al. [4] studied the local geometric structures of rank-one saddles that regulate reactions in systems with three or more dof. But a comprehensive theory of chemical reactions and efficient computational tools for reaction rate calculations in three or more dof systems which takes into consideration phase space structures still needs to be developed.

By merging tube dynamics of Koon el al. [6] and De Leon et al. [2] with Monte Carlo methods, ref. [5] provides some enabling theoretical and computational tools for the computation of lifetime distributions and rates of chemical reactions and scattering phenomena. The standard RRKM assumption of an unstructured phase space fails to account for the dynamics of systems exhibiting significant non-statistical behavior. This difficulty is overcome by using the homoclinic and heteroclinic intersection structure of tubes in phase space developed in ref. [6].

A main problem with the application of tube dynamics has been the computation of volumes of tube intersections in phase spaces of high dimension $[2,4]$. The paper [5] provides a starting point for overcoming this hurdle with an algorithm that uses tube dynamics to provide the initial bounding box for a Monte Carlo volume determination. We apply the method to a model-three-dof problem in which the hypotheses of TST do not hold: namely, the full-scattering of electrons of Rydberg hydrogen atoms in crossed electric and magnetic fields. We use a variety of methods and software that have been developed for tube dynamics $[6,7]$ to better understand the transport between different regions of phase space. The numerical results obtained are a demonstration of accurate lifetime distribution and rate calculations which overcome some of the difficulties of the standard statistical methods. Moreover, we mention some of our current effort in extending the applicability of our computational methods.

\section{Merge Tube Dynamics with Monte Carlo Methods}

Many chemical reactions proceed through energetic barriers. Such situations are well described in phase space where the energy-fixed hyper-surface determines different regions connected by the energy barriers, specifically by structures related to rank-one saddles associated with the barriers. To simplify the discussion, we consider a two-state system where one state is bound and the other is unbound. Fig. 1a shows a planar projection of the Hill region for the Rydberg atom in crossed fields; this Hill region shows the region in configuration space where the motion of the electron is possible for a given energy level.

The ionization of a Rydberg hydrogen atom in external crossed electric and magnetic fields has been studied by other authors (such as ref. [4]). To illustrate our method, we investigate the full-scattering problem, in which the system is prepared in an unbound initial state and we study the dynamics of formation of an excited 


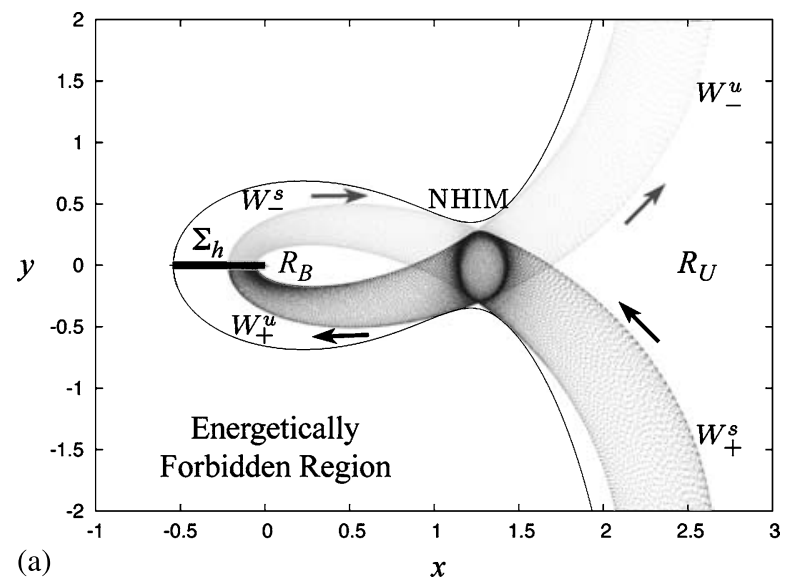

(a)

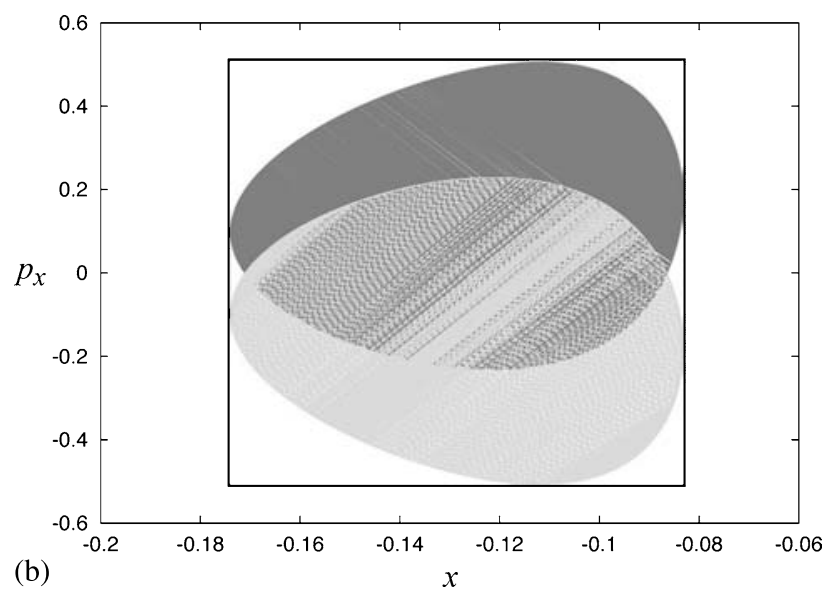

Fig. 1. a: Stable/Unstable $\left(W_{ \pm}^{s, u}\right)$ tubes of the NHIM in the bound $R_{B}$ and the unbound region $R_{U} . \Sigma_{h}$ denotes the Poincaré section. b: $x \dot{x}$ projection of the intersection of the high-dimensional tubes with the Poincaré section: the entrance (dark one) and the exit (light one)

Rydberg atom and its subsequent ionization. The dynamics of the outermost electron can be described by the following classical Hamiltonian,

$$
H=\frac{1}{2}\left(p_{x}^{2}+p_{y}^{2}+p_{z}^{2}\right)-\frac{1}{r}+\frac{1}{2}\left(x p_{y}-y p_{x}\right)+\frac{1}{8}\left(x^{2}+y^{2}\right)-\varepsilon x,
$$

where $r=\sqrt{x^{2}+y^{2}+z^{2}}$ is the distance from the electron to the center of the nuclear core and $\varepsilon$ is the scaled electric field strength.

For an energy value just above that of a saddle, the bottleneck near the saddle opens (see Fig. 1a) and the electron can move between the unbound and bound region. Specifically, in the bottleneck region, there exist 4 types of orbits: (i) a large number of bounded orbits which form a topological 3-sphere, i.e., instead of a periodic orbit $S^{1}$ in the 2dof case, we have an $S^{3}$ of bounded orbits in the 3dof case; it is an example of a normally hyperbolic invariant manifold (NHIM) where the stretching and contraction rates transverse to the NHIM dominate those tangent to the NHIM; (ii) 4 cylinders of asymptotic orbits that wind onto and off the NHIM; instead of a 2-dimensional invariant manifold in the 2dof case, one has a 4-dimensional invariant manifold: $S^{3} \times \mathbb{R}$ in a 5-dimensional energy manifold; they act as separatrices for the trajectories through the bottleneck region; (iii) those inside the tubes are transit trajectories which the electron must use to transit from one 
region to another; (iv) those outside the tubes are non-transit trajectories where the electron bounces back to its original region. These tubes are the geometric structures that completely control the reaction between the bound and unbound regions.

To study the reaction mechanism and compute its corresponding rates, we use carefully chosen 4-dimensional Poincaré sections $\Sigma_{h}$. Any trajectory from an unbound state to a bound state must be in the interior of the stable tube $W_{+}^{s}$ and continues in the interior of the unstable $W_{+}^{u}$ tube. $W_{+}^{s} \cup W_{+}^{u}$ which constitutes the capture reaction path from the unbound to bound state first pierces the Poincare section in the entrance or first Poincaré cut $\bar{C}_{+}^{1}$ (see Fig. 1b). Similarly, $W_{-}^{s} \cup W_{-}^{u}$ constitutes the escape reaction path, and any trajectory from the bound state to the unbound state has to pass through the exit or first Poincare cut $\bar{C}_{-}^{1}$ of this reaction path, just before reaction takes place. The intersection of the images of the entrance and the pre-images of the exit $f^{m-1}\left(\bar{C}_{+}^{1}\right) \cap f^{-(k-1)}\left(\bar{C}_{-}^{1}\right)$ under the Poincare map $f$ on $\Sigma_{h}$ are what give rise to full-scattering reactions. The problem can be simplified by looking only at the intersections of the images of the entrance with the exit itself: $\bar{C}_{+}^{l} \cap \bar{C}_{-}^{1}=f^{l-1}\left(\bar{C}_{+}^{1}\right) \cap \bar{C}_{-}^{1}$ where $l=m+k-1$. Any point inside the intersection
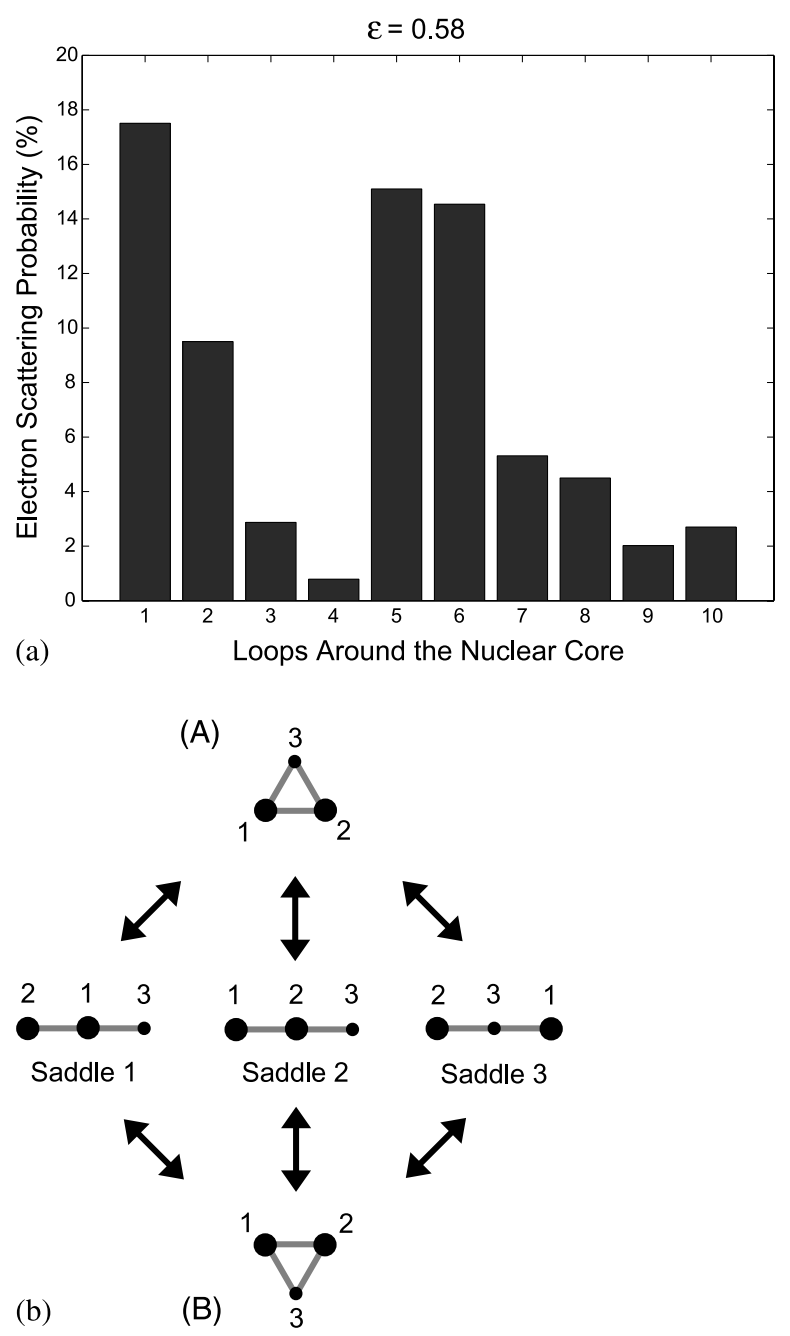

Fig. 2. a: The electron scattering lifetime distribution for the Rydberg atom. b: Isomerization of the modified $M_{3}$ cluster: a triatomic cluster of 2 heavy atoms and 1 light atom interacting through the pairwise Morse potential; the system has 2 permutationally distinct isomers, connected by 3 reaction pathways through 3 collinear saddles, each having the same potential barrier height 
$\bar{C}_{+}^{l} \cap \bar{C}_{-}^{1}$ is a trajectory that comes from the unbound state $R_{U}$, loops around the core and intersects $\Sigma_{h} l$ times before escaping to $R_{U}$.

Since the system is Hamiltonian, the Poincare map is volume preserving. Assuming a uniform distribution of incoming reactants on $\bar{C}_{+}^{1}$, then the fraction of products escaping after executing $m$ loops around the bound region is $V\left(\bar{C}_{+}^{m} \cap \bar{C}_{-}^{1}\right) / V\left(\bar{C}_{+}^{1}\right)$ where $V(A)$ is the volume of $A \subset \Sigma_{h}$. It is common to use Monte Carlo methods to compute numerically high-dimensional volumes. We first choose a hyper-rectangle "bounding box" containing the Poincaré cuts of the stable and unstable manifolds of the NHIM (see Fig. 1b). For the method to be efficient, it is important that this box contains as tightly as possible the Poincaré cuts. It is then easy to obtain an oracle that distinguishes whether randomly chosen points inside this box belong to the targeted object: e.g., a point belongs to the $m$-th overlap $\bar{C}_{+}^{m} \cap \bar{C}_{-}^{1}$ if it belongs to the exit $\bar{C}_{-}^{1}$ and its $(m-1)$-st backward iterate by the Poincare map belongs to the entrance. Given this oracle, we can use any standard Monte Carlo method to compute the desired volume.

In Fig. 2a, the percentage of reactants escaping from the bound state as a function of loops in the bound region is shown. The resulting scattering profile, computed from the 4D intersection volumes computed via the Monte Carlo method, is structured; i.e., it is not a simple exponential decay. The result implies that the phase space is not structureless, and we need to take into consideration the tube dynamics and non-RRKM effects in computing reaction rates.

\section{Current Work on Isomerization in a Tri-Atomic Molecule}

The method can be used in any system with rank-one saddles that separate phase space regions corresponding to different states of a chemical system, such as the isomerization of polyatomic clusters and bimolecular reactions. A primary concern is to develop a method that can be extended to higher-dimensional systems. In ref. [5], we construct the manifolds of the NHIM by computing an appropriate normal form of the Hamiltonian, which can be difficult in higher dimensions. Here, we outline some initial ideas that may be useful in extending the method that do not require the normal form (see ref. [8] for another method).

The key point is that to compute the hyper-volumes of intersection of invariant manifolds that give rise to the reaction rates and life-time distributions, one needs only to construct a hyper-rectangle "bounding box" in the $(2 n-2)$ space that contains the Poincaré cuts of the stable and unstable manifolds of the NHIM. Then a Monte-Carlo method can be used in this $(2 n-2)$-dimensional box to compute the volumes of interest. For energies close to that of a saddle, we can choose (i) the center of the box to be the intersection point of the stable (and unstable) manifold of the saddle point and the Poincare section, and (ii) the size of the box to be the smallest box that circumscribes the $(2 n-2)$-dimensional ball with radius $R=\beta \sqrt{2 \varepsilon / \min \left\{\omega_{k}\right\}}$, where $\varepsilon$ is the difference of the actual energy with that of the saddle, $\omega_{k}$ are the elliptic modes of the linear dynamics at the saddle - recall that the quadratic normal form at the saddle is given by

$$
H_{2}=\lambda q_{1} p_{1}+\sum_{2}^{n} \frac{\omega_{k}}{2}\left(q_{k}^{2}+p_{k}^{2}\right)
$$


- and $\beta>1$ is a factor of enlargement, to ensure that the box contains the targeted object. We start with, e.g., $\beta=2$, compute the volume of interest, and then increase $\beta$ and recompute the same volume; if the difference of these two volumes is smaller than our numerical error tolerance, we take this $\beta$; if not, we restart the process with a larger $\beta$ as an initial guess. This iterative process allows us to compute the intersection volumes of the stable and unstable manifolds of the NHIM, and therefore the reaction rate.

Currently we are applying this method to the modified $M_{3}$ cluster which has been introduced in ref. [9] to study the mass effect in isomerization dynamics of triatomic molecules (see Fig. 2b). Despite the same flux across each dividing surface, a significant difference arises among the possibilities of occurrence of reactions through respective pathways. These observations are symptoms of breakdown of the statistical hypothesis behind TST, and more detailed investigation of transport mechanisms along the reaction paths is necessary. For multi-channel reactions such as isomerization of the modified $M_{3}$, the heteroclinic intersection structure is also needed; techniques for tube dynamics developed in ref. [6] are useful. To study the structure of these intersections, the choice of a suitable set of Poincare sections is important. The computation of the volumes of all these different intersections via the bounding box method is a key step in computing the reaction rates between the two isomers following different reaction channels.

Acknowledgement. NSF-ITR grant ACI-0204932, ICB-ARO grant DAAD19-03-D-0004, NSF-DMS0505711, Fulbright-GenCat postdoc fellowship, MCyT/FEDER grant BFM2003-07521-C02-01, NSF postdoc fellowship NSF-DMS 0402842, JSPS Research Fellowships.

\section{References}

1. De Leon, N.: J. Chem. Phys. 96, 285 (1992)

2. De Leon, N., Mehta, M. A., Topper, R. Q.: J. Chem. Phys. 94, 8310 (1991); 94, 8329 (1991)

3. Komatsuzaki, T., Berry, R. S.: Proc. Natl. Acad. Sci. USA 98, 7666 (2001)

4. Uzer, T., Jaffé, C., Palacián, J., Yanguas, P., Wiggins, S.: Nonlinearity 15, 957 (2002)

5. Gabern, F., Koon, W. S., Marsden, J. E., Ross, S. D.: Physica D211, 391 (2005)

6. Koon, W. S., Lo, M. W., Marsden, J. E., Ross, S. D.: Chaos 10, 427 (2000)

7. Gómez, G., Koon, W. S., Lo, M. W., Marsden, J. E., Masdemont, J., Ross, S. D.: Nonlinearity 17, 1571 (2004)

8. Dellnitz, M., Grubits, K., Marsden, J. E., Padberg, K., Thiere, B.: Reg. Chaot. Dyn. 10, 173 (2005)

9. Yanao, T., Koon, W. S., Marsden, J. E.: Few-Body Systems 38, 161 (2006) 